\title{
Process Optimization for Preparation of an Antibacterial Agent Using a Combined Extract of Tulsi and Marigold Leaves
}

\author{
Shalini Paul, Koel Mukherjee, Amrita De, Aparupa Bhattacharyya, \\ Srabanti Basu \\ Department of Biotechnology, Heritage Institute of Technology, India
}

\begin{abstract}
Medicinal plants and herbs contain substances known to modern and ancient civilizations for their healing properties. India is rich in biodiversity and many of its plants are known to have medicinal properties. This paper highlights two tropical and subtropical important plants -- Ocimum sanctum (tulsi) and Targetes erecta (marigold) due to their easy availability and antimicrobial effectiveness. Several studies have been done on the antimicrobial properties of the individual extracts; however, few attempts have been made to study their antimicrobial properties in combination. The present work attempts to prepare an antimicrobial agent possessing the maximum efficiency, with a combination of leaf extracts from tulsi and marigold prepared in the most suitable solvent. Objective of the present work is to optimize the preparation method of the antimicrobial product and to check the properties of the product. Propylene glycol was found to be the most suitable extraction solvent. Process for preparation of the antimicrobial agent was then optimized by Response Surface Methodology using Design Expert Software 10. The extract retained its $72 \%$ and $62 \%$ of its antibacterial activity after 35 days when preserved at $4{ }^{\circ} \mathrm{C}$ against E.coli and $S$. aureus respectively.
\end{abstract}

Keywords: Tulsi, marigold, antimicrobial properties, Response Surface Methodology

\section{Introduction}

Since ancient times, there has been a continuous effort in finding healing powers in plants. However, for the past few decades, the antimicrobial agents obtained from bacterial and fungal sources have limited the use of the ones obtained from higher plants. The interest in antimicrobial agents from plants has been restored, mainly for two reasons. First, microbes are becoming drug-resistant and traditional methods of antibiotic development failed to keep pace with the development of drug resistance in bacteria. Second, people are becoming more aware of the ill effects of overuse of antibiotics [1].

Food safety is a global problem as many people die from food borne diseases worldwide. According to WHO, at least 2 billion people get ill due to consumption of unsafe food. Thermal processing is a common method to destroy microorganisms present in food but the method can destroy some nutrients. Use of preservatives is another common method to reduce the risk of illness. But increasing regulatory restrictions and negative response from consumers to the use of chemical preservatives have limited their use and developed a need for new antimicrobial agents [2].

It is estimated that plant materials are either present in, or have provided the models for $50 \%$ Western drugs $[3,4]$. The primary benefits of using plant derived medicines are that they are relatively safer than synthetic alternatives, offering profound therapeutic benefits and more affordable treatment. Though most of the clinically used antibiotics are produced by soil microorganisms or fungi, higher plants have also been a source of antibiotics. Examples of these are the bacteriostatic and anti-fungicidal properties of Lichens, the antibiotic action of allinine in Allium sativum (garlic), or the antimicrobial action berberines in goldenseal (Hydrastis canadensis) [5].

Medicinal plants and herbs contain substances known to modern and ancient civilizations for their healing properties. Until the development of chemistry and, particularly, the synthesis of organic compounds in the 19th century, medicinal plants and herbs where the sole source of active principles capable of curing man ailments. In more recent history, the use of plants as medicines has involved the isolation of active compounds, beginning with the isolation of morphine from opium in the early 19th century [6, 7]. India is rich in biodiversity and many of its plants are known to have medicinal properties. This paper highlights two tropical and subtropical important plants -- Ocimum sanctum (tulsi) and Targetes erecta (marigold). The reasons behind the selection of these plants can be attributed to their easy availability and medicinal effectiveness. Several studies have been done on the antimicrobial properties of the individual extracts; however, few attempts have been made to study their antimicrobial properties in combination. The present work attempts to prepare an antimicrobial product possessing the maximum efficiency, with a combination of extracts from tulsi and marigold prepared in the most suitable solvent. Objective of the present work is to optimize the preparation method of the antimicrobial product and to check the properties of the product. 


\subsection{Collection of samples}

\section{Materials and Methods}

Fresh leaves of tulsi and marigold were collected from adult plants available in the institute garden. The leaves were preserved in refrigerator for a maximum period of three days.

\subsection{Selection of solvent}

Fresh plant leaves were taken for preparation of $10 \%$ extract (w/v) in Ethanol, Methanol, Acetone, Propylene glycol (PPG) and water. The antimicrobial activities of the plants were tested against 24-h old cultures of laboratory microbial strains of Bacillus subtilis, and Escherechia coli by measuring the zone of inhibition. The inoculums were prepared in nutrient broth at $37^{\circ} \mathrm{C}$ having an absorbance of 0.6-0.8 at $600 \mathrm{~nm}$. The cultures were maintained in the respective media in agar slant at $15^{\circ} \mathrm{C}$ with transfer to fresh media after 7 days. Minimum inhibitory concentration (MIC) was determined for each extract to find out the most effective solvent.

\subsection{Optimization of the procedure for the preparation of an anti-microbial agent using a combination of tulsi and marigold extracts}

The process for preparation of extract was optimized with the most suitable solvent by Response Surface Methodology using Design Expert Software (Version 10). Central composite design model was chosen for optimization of the process. The design consists of three distinct sets of experimental runs:

1. A factorial design in the factors studied

2. A set of center points, experimental runs whose values of each factor are the medians of the values used in the factorial portion. This point is often replicated in order to improve the precision of the experiment;

3. A set of axial points, experimental runs identical to the centre points except for one factor, which will take on values both below and above the median of the two factorial levels, and typically both outside their range. All factors are varied in this way [8].

In this process three numeric factors have been taken as independent factor viz. mass of marigold ( $\mathrm{g}$ ), mass of tulsi $(\mathrm{g})$ and extraction time $(\mathrm{h})$. These three factors were varied in the following manner:

Mass of marigold $=5-20 \mathrm{~g}$

Mass of tulsi $=5-20 \mathrm{~g}$, Extraction time $=2-24 \mathrm{~h}$

Growth inhibition was measured by checking the absorbance of the culture before and after incubation with the extract. Percentage of growth inhibition was taken as the dependent factor or response.

\subsection{Phytochemical analysis of the optimized product}

The antimicrobial extract prepared under the optimized conditions was used for qualitative analysis of phytochemicals. Alkaloids, saponins, phytosterols, phenols, flavonoids and diperpenes, were tested following standard protocols [9].

2.4.1 Detection of alkaloids: Few drops of hydrochloric acid were added to the extract and the mixture was filtered. A portion of the filtrate was treated with Mayer's reagent (potassium mercuric iodide) to detect alkaloid, indicated by the formation of yellow colored precipitate. Another portion of the filtrate was treated with Wagner's reagent (iodine in potassium iodide). Formation of brown/reddish precipitate indicates the presence of alkaloids.

2.4.2 Detection of saponins: Saponin was detected by foam test. $0.5 \mathrm{gm}$ of extract was shaken with $2 \mathrm{ml}$ of water. If foam produced persists for ten minutes, it indicates the presence of saponins.

2.4.3 Detection of phytosterols: Phytosterols were detected by Salkowski's Test. The extract was treated with chloroform and filtered. The filtrate was treated with few drops of concentrated sulfuric acid, shaken and allowed to stand. Appearance of golden yellow colour indicates the presence of triterpenes.

2.4.4 Detection of phenols: For detection of phenol, the extract was treated with 3-4 drops of ferric chloride solution. Formation of bluish black colour indicates the presence of phenols.

2.4.5 Detection of flavonoids: Flavonoids were detected by alkaline reagent test and lead acetate test. For alkaline reagent test, the extract was treated with few drops of sodium hydroxide solution. Formation of intense yellow colour, which becomes colourless on addition of dilute acid, indicates the presence of flavonoids. For the lead acetate test, the extract was treated with few drops of lead acetate solution. Formation of yellow colour precipitate indicates the presence of flavonoids. 
2.4.6 Detection of diterpenes: The extract was added to water and treated with 3-4 drops of copper acetate solution. Formation of emerald green colour indicates the presence of diterpenes.

\subsection{Shelf life screening}

The extract was preserved at the room temperature (average $25^{\circ} \mathrm{C}$ ) and in the refrigerator $\left(4^{\circ} \mathrm{C}\right.$ ). Shelf life analysis was carried out by testing the antimicrobial activity against the three bacterial strains - B.subtilis, E.coli and Staphylococcus aureus used in this study. The antimicrobial activity was tested at an interval of 15 days, starting from the 5th day and ending at the 35th day.

\subsection{Selection of Solvent:}

\section{Results And Discussions}

Both tulsi and marigold extracts showed different levels of antimicrobial activities in different solvents. Best inhibitory effect was observed with propelene glycol (PG) extract for both tulsi and marigold. PG was selected as the solvent for preparation of an antimicrobial agent using a combination of tulsi and marigold extracts.

Table 1 Antibacterial activity of tulsi and marigold extract prepared in different solvents

\begin{tabular}{|l|l|l|l|l|l|l|}
\hline Solvent & \multicolumn{4}{l}{ Zone of inhibition (mm) against B. subtilis } & \multicolumn{4}{l|}{ Zone of inhibition (mm) against E. coli } \\
\hline & Only solvent & Tulsi & Marigold & Only solvent & Tulsi & Marigold \\
\hline Methanol & 1 & 11 & 18 & 1 & 6 & 10 \\
\hline Ethanol & 2 & 13 & 11 & 2 & 19 & 12 \\
\hline Acetone & 0 & 0 & 10 & 0 & 0 & 0 \\
\hline Water & 0 & 0 & 0 & 0 & 0 & 0 \\
\hline PG & 0 & 15 & 20 & 0 & 21 & 15 \\
\hline
\end{tabular}

3.2 Optimization of the procedure for the preparation of an anti-microbial agent using a combination of tulsi and marigold extracts:

The experimental design as suggested by Design Expert Software along with the values of responses is presented in TABLE 2.

Table 2. Experimental design predicted by the Design Expert Software for antimicrobial activity of combined tulsi-marigold extract on B. subtilis and E. coli culture

\begin{tabular}{|c|c|c|c|c|c|}
\hline Run & $\begin{array}{l}\text { Factor1 (mass of } \\
\text { marigold) }(\mathrm{g})\end{array}$ & $\begin{array}{l}\text { Factor2 (mass } \\
\text { of tulsi) (g) }\end{array}$ & $\begin{array}{l}\text { Factor3 (extraction } \\
\text { time) (hours) }\end{array}$ & $\begin{array}{l}\text { Response (\% inhibition) } \\
\text { For B. subtilis }\end{array}$ & $\begin{array}{l}\text { Response }(\% \\
\text { inhibition) For E. coli }\end{array}$ \\
\hline 2 & 12.5 & 12.5 & 13 & 96.95 & 97.34 \\
\hline 4 & 12.5 & 12.5 & 13 & 97.28 & 97.21 \\
\hline 5 & 25.1134 & 12.5 & 13 & 89.04 & 88.24 \\
\hline 6 & 20 & 20 & 2 & 79.67 & 78.98 \\
\hline 8 & 0 & 12.5 & 13 & 86.6 & 85.61 \\
\hline 9 & 12.5 & 12.5 & 31.4997 & 87.65 & 86.69 \\
\hline 10 & 5 & 20 & 24 & 96.62 & 89.23 \\
\hline 11 & 12.5 & 0 & 13 & 91 & 87.65 \\
\hline 12 & 5 & 20 & 2 & 75.88 & 71.27 \\
\hline 13 & 12.5 & 12.5 & 13 & 96.18 & 96.18 \\
\hline 17 & 20 & 5 & 24 & 88.85 & 89.76 \\
\hline 18 & 20 & 20 & 24 & 91.98 & 90.45 \\
\hline 19 & 12.5 & 12.5 & 13 & 94.56 & 96.78 \\
\hline 20 & 12.5 & 12.5 & 13 & 94.06 & 97.6 \\
\hline
\end{tabular}

Fig. 1 (A) and (B) represents the combined effect of mass of tulsi and mass of marigold on growth inhibition of B. subtilis and E. coli respectively when extraction time was kept constant at $13 \mathrm{~h}$. Same trends were observed in both the graphs. Growth inhibition increased with individual increase in mass of tulsi and mass of marigold. However, the inhibition decreases after a certain point when the mass of tulsi and marigold had been increased together. It may be due to the fact that the active chemicals become highly concentrated with increase in mass of both tulsi and marigold. They may not be able to reach cells effectively at high concentrations. It may be also possible that the active chemicals get partially inactivated due to their interaction in the concentrated extracts. 

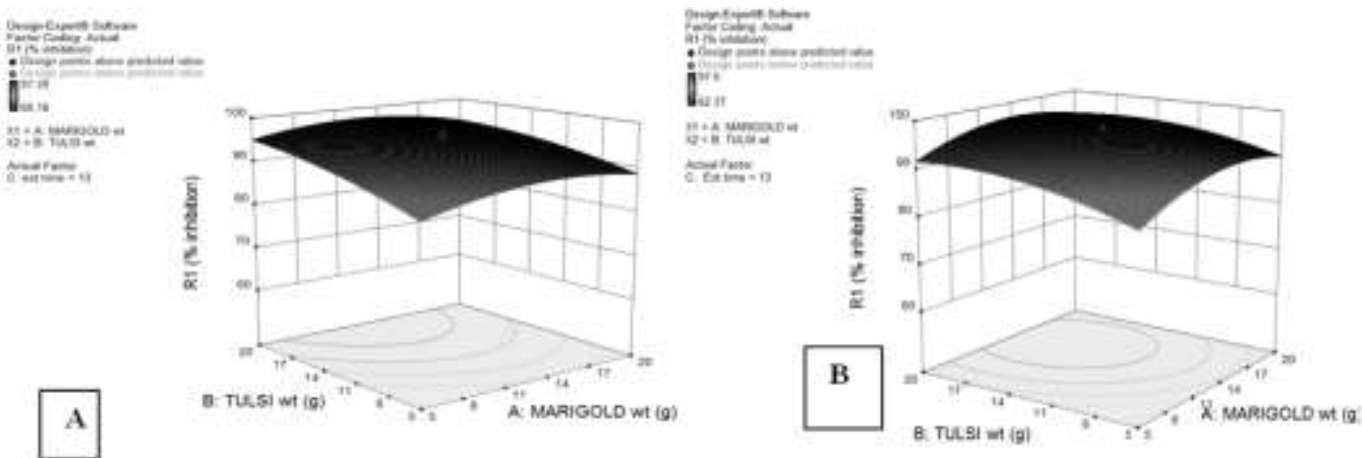

Fig. 1 Graphical Representation Of Growth INHIBITION ON (A) B. Subtilis AND (B) E. Coli With VARYING MASS OF MARIGOLD AND TULSI

Fig. 2 (A) and (B) represents the combined effect of extraction time and mass of marigold on growth inhibition of B. subtilis and E. coli respectively when the mass of tulsi was kept constant at $12.5 \mathrm{~g}$. In both the cases, growth inhibition increased with increase in extraction time upto a certain point and after that it decreased. This may be the fact that with the increase in extraction time some compound may be degraded. Due to this after achieving the optimum value inhibition decreased.

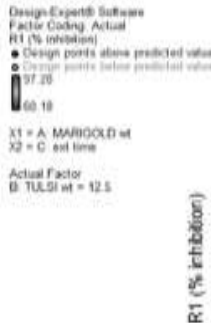

A
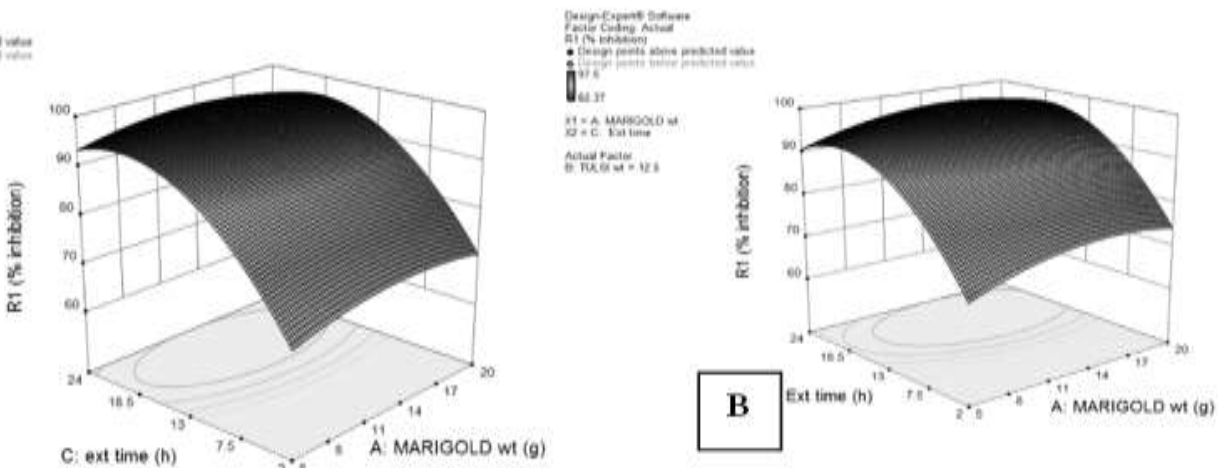

Fig. 2 GRAPHICAL REPRESENTATION OF GROWTH INHIBITION ON (A) B. SUBTILIS AND (B) E. COLI WITH VARYING EXTRACTION TIME AND MASS OF MARIGOLD

Combined effect of extraction time and mass of tulsi on growth inhibition followed the same trend as above. Fig. 3 (A) and (B) represent the result.
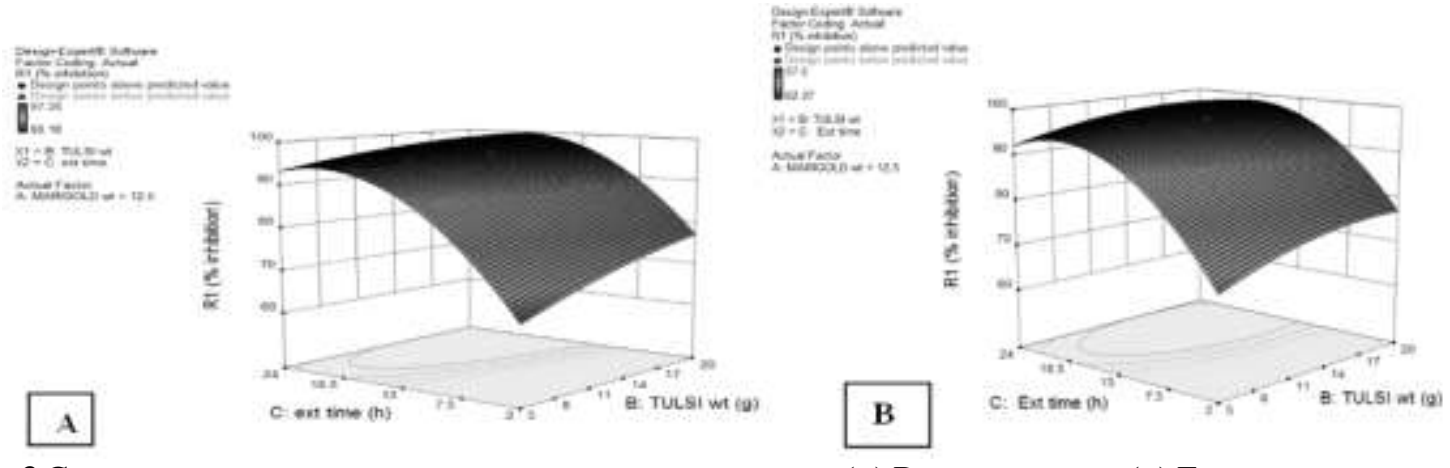

FIG. 3 GRAPHICAL REPRESENTATION OF GROWTH INHIBITION ON (A) B. SUBTILIS AND (B) E. COLI WITH VARYING EXTRACTION TIME AND MASS OF TULSI

Regression analysis was done with the obtained data and it was found that the quadratic model fits the data both for B. subtilis and E. coli. Equations in terms of actual factors for B. subtilis and E. coli are given below.

For B.subtilis

\%Inhibition $=38.92601+1.58961 \times$ MarigoldWt $+1.64761 \times$ TulsiWt $+3.84350 \times$ Time

$-0.015111 \times$ MarigoldWt $\times$ TulsiWt $-7.27273 \times 10^{-3} \times$ MarigoldWt $\times$ Time

$-0.027788 \times$ TulsiWt $\times$ Time $-0.048553 \times$ MarigolWt $^{2}-0.023189 \times$ TulsiWt $^{2}-0.089303 \times$ Time $^{2}$ 
For E.coli

$$
\begin{aligned}
& \text { \% Inhibition }=38.60560+2.11437 \times \text { MarigolWt }+1.82858 \times \text { TulsiWt }+3.56240 \times \text { Time } \\
& -0.011667 \text { MarigoldWt } \times \text { TulsiWt }-6.10606 \times 10^{-3} \text { MarigoldWt } \times \text { Time }-0.017227 \times \text { TulsiWt } \times \text { Time } \\
& -0.064683 \text { MarigoldW } t^{2}-0.043814 \text { TulsiWt }{ }^{2}-0.089875 \times \text { Time }^{2}
\end{aligned}
$$

The optimized conditions for preparation of an antibacterial agent using tulsi and marigold extract are: mass of tulsi: $12.5 \mathrm{~g}$; mass of marigold: $12.5 \mathrm{~g}$, extraction time: $13 \mathrm{~h}$

\subsection{Phytochemical Analysis:}

TABLE 3 shows presence of different pytochemicals in the combined extract of tulsi and marigold prepared under optimum conditions. The extract showed positive results for alkaloids, phytosterols, phenols, flavonoids and diterpenes.

TABLE 3. Presence of phytochemicals in the combined extract of tulsi and marigold prepared under optimum conditions

\begin{tabular}{|l|l|l|l|l|l|}
\hline ALKALOIDS & SAPONINS & PHYTOSTEROLS & PHENOLS & FLAVONOIDS & DITERPENES \\
\hline+ & -- & + & + & + & + \\
\hline
\end{tabular}

\subsection{Shelf life Analysis:}

Antibacterial activity of the combined extract of tulsi and marigold prepared under optimum condition was analsyed against B. subtilis and E. coli. It was observed that the extract had retained its $72 \%$ of its antibacterial activity after 35 days when preserved at $4^{\circ} \mathrm{C}$. The antibacterial activity against B. subtilis after 20 days and 45 days was much less compared to E. coli. However, the extract showed retention of $62 \%$ antibacterial activity when tested against S. aureus.

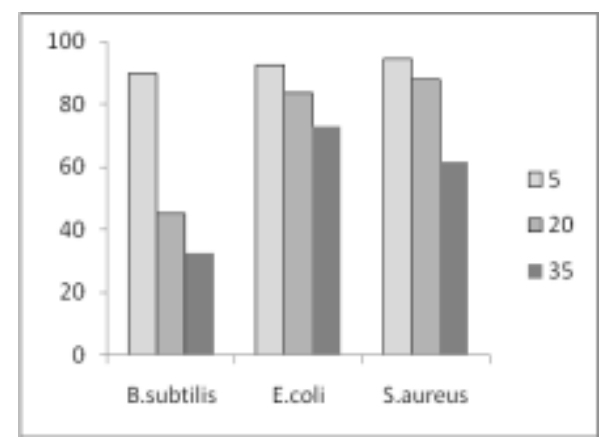

FIG. 4 ANTIB ACTERIAL ACTIVITY OF THE COMBINED EXTRACT OF TULSI AND MARIGOLD PRESERVED AT $4^{\circ} \mathrm{C}$ AFTER 5, 20 AND 35 DAYS

\section{Conclusion}

The tulsi- marigold extract, prepared under the optimized conditions of $12.5 \mathrm{~g}$ of tulsi, $12.5 \mathrm{~g}$ of marigold and an extraction time period of $13 \mathrm{~h}$ was found to be quite effective with $72 \%$ of its antibacterial activity retained after 35 days when preserved at $4^{\circ} \mathrm{C}$. Therefore, it can be concretely concluded that the prepared extract, with its high antimicrobial activity, has the potential to be implemented in the production of different antiseptic agents, food preservatives or organic pesticides.

\section{References}

[1]. A C Abreu, A J McBain and M Simoes M, Plants as sources of new-antimicrobials and resistance-modifying agents, Natural Product Reports, 9, 2012, 1007-1021.

[2]. K K Di R Matthews and T Hintz, The use of plant antimicrobial compounds for food preservation, Biomed Research International, 2015, doi.org/10.1155/2015/246264

[3]. J E Robbers, M K Speedie and V E Tyler, Pharmacognosy and pharmacobiotechnology (Williams \& Wilkins, Baltimore, 1996).

[4]. A S Salhab, A A Issa and I Alhougog, On the contraceptive effect of castor beans, International Journal of Pharmacognosy, 34 , 1996, 1-3.

[5]. M M Cowan, Plant products as antibacterial agents, Clinical Microbiology Review, 12, 1999, 564-582.

[6]. A D Kinghorn, Pharmacognosy in the 21st century, Journal of Pharmacy and Pharmacology, 53, 2001, 135-148.

[7]. G Samuelsson, Drugs of natural origin: A textbook of pharmacognosy (5th Swedish Pharmaceutical Press, Stockholm, 2004).

[8]. R H Myers,Response Surface Methodology (Boston: Allyn and Bacon, Inc., 1971).

[9]. P Tiwari, B Kumar, M Kaur, G Kaur and H Kaur. Phytochemical screening and Extraction: A Review, Internationale Pharmaceutica Sciencia, 1(1), 2011, 98-106 